\title{
Efficacy and safety of subcutaneous administration of lyophilized powder of alfa-erythropoietin to maintain hemoglobin concentrations among hemodialysis patients
}

This article was published in the following Dove Press journal: International Journal of Nephrology and Renovascular Disease 20 September 2017

Number of times this article has been viewed

\section{Bancha Satirapoj \\ Rattanawan Dispan \\ Ouppatham Supasyndh}

Division of Nephrology, Department of Medicine, Phramongkutklao Hospital and College of Medicine, Bangkok, Thailand
Correspondence: Bancha Satirapoj Division of Nephrology, Department of Medicine, Phramongkutklao Hospital and College of Medicine, 315 Rachavitee Road, Bangkok 10400, Thailand

Tel/Fax +6626444676

Email satirapoj@yahoo.com
Background: Anemia associated with chronic kidney disease (CKD) often requires treatment with recombinant human erythropoietin (EPO). This study investigated the therapeutic equivalence between lyophilized powder and standard liquid EPO alfa by subcutaneous (SC) administration in hemoglobin maintenance among patients on hemodialysis.

Methods: This was a single-blinded, randomized, controlled, single-center, parallel-group study regarding the treatment of anemia among CKD patients on hemodialysis and being treated with stable doses of EPO alfa at least for 12 weeks. Anemic hemodialysis patients $(n=63)$ received standard liquid or lyophilized powder EPO alfa for 24 weeks by SC administration. Achievement of the target hemoglobin concentration and safety and tolerability end points were documented. Results: Baseline mean hemoglobin level was $11.1 \pm 0.7 \mathrm{~g} / \mathrm{dL}$ using lyophilized powder EPO alfa and $11.2 \pm 0.9 \mathrm{~g} / \mathrm{dL}$ using standard liquid EPO alfa. The baseline median dose of EPO alfa was 126.4 (interquartile range [IQR] 81.6-163.6) U/kg/week in the lyophilized powder EPO alfa group and 116.9 (IQR 76.5-144.1) U/kg/week in the standard liquid EPO alfa group. Treatment with SC lyophilized powder EPO alfa maintained mean hemoglobin and hematocrit concentrations after switching from standard liquid EPO alfa. No statistical significance between groups was reported for hemoglobin concentrations and weekly dose of EPO alfa during the study. No safety concerns were raised, including positive anti-EPO antibodies.

Conclusion: In this study of anemia therapy among patients with end-stage renal disease on hemodialysis therapy, the SC injection of lyophilized powder EPO alfa was well tolerated and effectively maintained hemoglobin levels. Future studies of larger size and longer duration will be required to assess safety profiles.

Keywords: anemia, hemodialysis, lyophilized powder, erythropoietin, subcutaneous injection

\section{Background}

Chronic kidney disease (CKD) is frequently associated with anemia. ${ }^{1}$ A direct relationship has been observed between the severity of the anemia and the decline in kidney function, and the main cause of anemia is attributed to diminished erythropoietin (EPO) production. ${ }^{2,3}$ Correction of anemia and maintenance of stable hemoglobin levels using recombinant human EPO is an important aspect of CKD treatment. ${ }^{4,5}$ Treatment with recombinant human EPO has been shown to be highly effective in terms of correcting anemia, improving the quality of life and decreasing the need for red blood cell transfusions in the majority of CKD patients with and without dialysis. ${ }^{6-8}$

The active ingredient in HEMAX is lyophilized powder of EPO alfa, a 165-aminoacid glycoprotein produced by recombinant DNA technology in genetically modified 
mammal cells. Its molecular weight is $30.4 \mathrm{kDa}$ and its amino acid structure is identical to that of natural EPO. The plasma half-life concentration is achieved between 4 and 13 hours following subcutaneous (SC) administration. The lyophilized powder will immediately dissolve in solution, and the protein solution can be stored at $2-8^{\circ} \mathrm{C}$ for up to 1 week. In an initial study, injecting mice with the EPO-loaded poly (lactic-co-glycolic acid) microspheres or lyophilized powder of EPO resulted in rising hemoglobin level, and the mice did not develop anti-EPO IgG antibodies. ${ }^{9}$ Several clinical trials have confirmed the ability of lyophilized powder EPO alfa to induce effective erythropoiesis, ${ }^{10-12}$ but, there was limited study of lyophilized powder EPO alfa among dialysis patients. The economical benefit is also appreciated in Thailand because the cost per unit of lyophilized powder EPO alfa preparation is lower than that of standard liquid EPO alfa. Here, we report the results assessing the hemoglobin response and safety of a 24-week SC administration of lyophilized powder EPO alfa among patients on hemodialysis and treated with stable doses of standard reference EPO product.

\section{Methods}

\section{Study design}

This single-center, single-blinded, randomized, controlled, parallel-group study evaluated the efficacy and safety of SC injection of lyophilized powder EPO alfa among anemic hemodialysis patients. Patients were followed up for assessments for 24 weeks after the treatment period. The study protocol was approved by the Ethics Committee of Institutional Review Board of Royal Thai Army Medical Department, Bangkok, Thailand, and the study was conducted as per the Declaration of Helsinki. Written informed consent was obtained from all patients at screening.

Trial registration number is TCTR2016121400 and was retrospectively registered on 13 December 2016.

The primary objective was to determine the hemoglobin levels and total weekly EPO doses between SC treatment with lyophilized powder and standard liquid EPO alfa among anemic hemodialysis patients. The secondary objectives were to assess the effects on markers of erythropoiesis including iron mobilization markers (total iron-binding capacity, transferrin saturation, serum iron and serum ferritin) and other metabolic markers (serum albumin, urea nitrogen, creatinine, parathyroid hormone (PTH), calcium and phosphorus).

\section{Study population}

Eligibility criteria included male and female patients aged 18-85 years diagnosed with CKD receiving hemodialysis who presented stable hemoglobin levels of 10-11.5 g/dL with standard liquid EPO alfa product (EPREX, Cilag AG, Schaffhausen, Switzerland) treatment for at least 12 weeks. All patients had adequate dialysis by a single pool Kt/Vurea greater than 1.2 per dialysis treatment and adequate iron status ( $\geq 20 \%$ of transferrin saturation and $\geq 200 \mathrm{ng} / \mathrm{mL}$ of ferritin). Patients were excluded from the study if they were on peritoneal dialysis or had identified causes of anemia such as gastrointestinal hemorrhage, hematologic malignancy, chronic infection and nutritional anemia. In addition, patients were excluded if they were hyporesponsive to EPO (defined as an intravenous EPO dose of $>360 \mathrm{IU} / \mathrm{kg}$ weekly or an intravenous darbepoetin dose of $>1.8 \mathrm{mg} / \mathrm{kg}$ weekly) within the prior 12 weeks.

\section{Interventions}

All eligible patients were randomized using block randomization with codes concealed in sequentially numbered opaque envelopes. Patients were randomly assigned to either the same dose of the standard liquid EPO alfa (EPREX, Cilag AG) or the lyophilized powder EPO alfa (HEMAX, Bio Sidus S.A., Buenos Aires, Argentina) by SC administration for 24 weeks. The total weekly dosage was divided into one to three times followed by the hemodialysis session. The hemoglobin concentration was maintained within the target range of $10-11.5 \mathrm{~g} / \mathrm{dL}$ by adjusting the dose of EPO alfa. Oral iron supplementation was permitted throughout the study to maintain ferritin levels between 50 and $300 \mathrm{ng} / \mathrm{mL}$, and intravenous iron supplementation was only allowed for patients who were intolerant of oral iron.

\section{Assessments of outcomes}

Eligible patients were scheduled every 4 weeks to monitor hemoglobin, hematocrit, reticulocyte count, iron status, dialysis dose $(\mathrm{Kt} / \mathrm{V})$, blood pressure and clinical status. Midweek collection of pre-dialysis blood samples was measured to create a serum albumin and hematology panel. Quality of life was prospectively measured using the SF-36 questionnaire with physical and mental health-specific outcomes at baseline and at the end of the study.

Safety was assessed by physical examinations, clinical laboratory tests and the incidence and severity of adverse events recorded from treatment onset through follow-up. Anti-EPO antibodies were determined at baseline and at the end of the study. Serum patients were assayed for anti-EPO antibody detection by enzyme-linked immunosorbent assay (MyBiosource Inc, San Diego, CA, USA) to detect the presence of antibodies to EPO quantitatively. 


\section{Statistical analysis}

Data were expressed as mean \pm standard deviation and median with interquartile range (IQR). Comparisons between treatment groups were carried out using the Independent $t$-test, Mann-Whitney $U$ test and chi-square test. Comparisons within groups were conducted by repeated measures analysis of variance using SPSS software (SPSS version 14.0, Chicago, IL, USA). Statistical significance was determined as a $p$-value less than 0.05 .

\section{Results}

\section{Demographic characteristics}

In all, 67 hemodialysis patients were enrolled and randomly assigned; 63 (94\%) completed protocols through followup at week 24 . The primary reasons for withdrawing from the study were protocol deviations and withdrawn consent. Patient disposition for both treatment groups are presented in Figure 1. All patients remained 100\% adherent to the EPO prescription based on SC injection counts. Baseline demographics of patients for both groups are shown in Table 1. Patients were generally compliant with the study medication and SC injection in both arms, as assessed by documented $\mathrm{SC}$ injection counts. No patient received a blood transfusion during the study. No significant differences in sex, age, body weight, body mass index, duration of dialysis and comorbid diseases were noted between the two groups (Table 1).

\section{Hemoglobin and hematocrit}

Mean baseline hemoglobin and hematocrit concentrations were similar across the treatment groups, ranging from 10.4 to $12.1 \mathrm{~g} / \mathrm{dL}$ and 31.1 to $36.8 \%$, respectively. There were no significant differences in mean hemoglobin and hematocrit levels at any time point during 24 weeks between the lyophilized powder of EPO alfa-treated patients and the standard liquid EPO alfa-treated patients (Table 2 and Figure 2).

Mean weekly EPO alfa doses for both groups are shown in Table 3. The baseline mean EPO alfa dose requirements for hemoglobin level maintenance were 126.4 (IQR 81.6-163.6) $\mathrm{U} / \mathrm{kg} /$ week and 116.9 (IQR 76.5-144.1) U/kg/week in the standard liquid EPO alfa group. No significant differences were observed in weekly doses over time between the two groups.

\section{Iron metabolism and use}

Mean baseline levels at various time points during 24 weeks for serum ferritin, transferrin, transferrin saturation, total iron binding capacity and serum iron concentrations are shown in Table 3. Although a high degree of variability was noted in iron parameters, no significant difference was observed in transferrin saturation and ferritin level between the two groups (Table 3).

\section{Metabolic profiles}

At baseline, no significant difference was found in blood pressure, blood urea nitrogen, serum creatinine, albumin, serum calcium, phosphate and intact PTH between the two groups. No requirement was noted for an increase in antihypertensive treatment compared with standard liquid EPO alfa. Small increases in intact PTH were noted for the standard liquid EPO alfa group. Serum creatinine levels

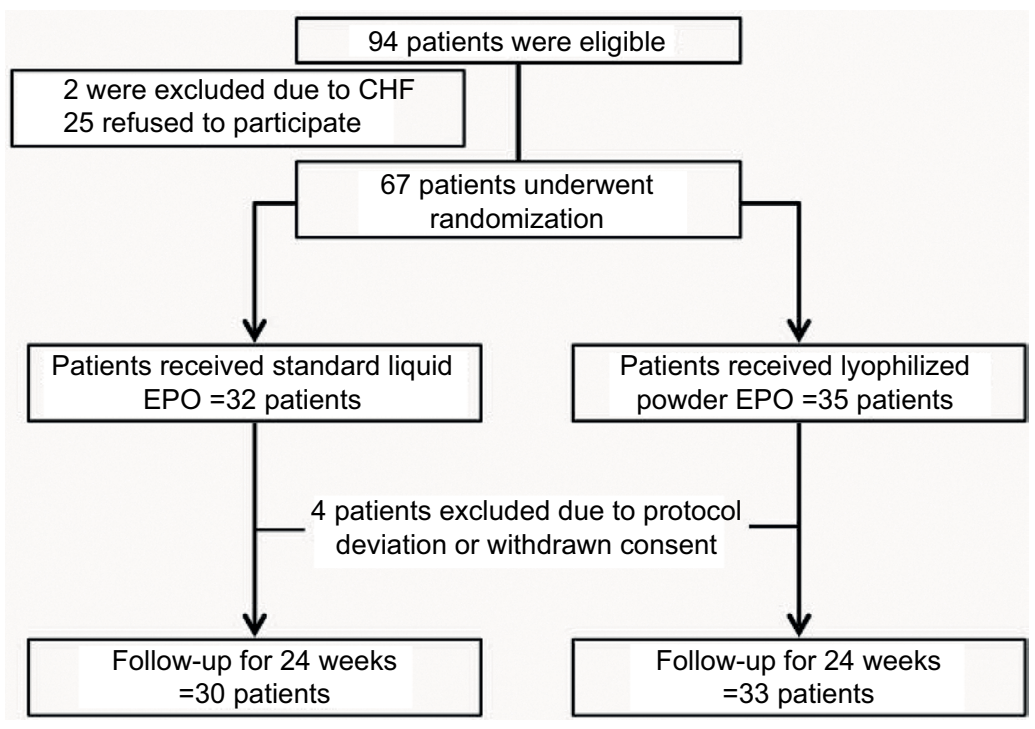

Figure I Flowchart of the selection of patients for the study. Abbreviations: $\mathrm{CHF}$, congestive heart failure; EPO, erythropoietin. 
Table I Baseline demographic characteristics

\begin{tabular}{llll}
\hline & $\begin{array}{l}\text { Standard liquid EPO } \\
(\mathbf{n = 3 0})\end{array}$ & $\begin{array}{l}\text { Lyophilized powder EPO } \\
(\mathbf{n = 3 3 )}\end{array}$ & p-value \\
\hline Male, $\mathrm{n}(\%)$ & $16(53.3)$ & $18(54.5)$ & 0.923 \\
Age (years) & $58.47 \pm 14.19$ & $57.42 \pm 10.84$ & 0.743 \\
Duration of dialysis (months) & $30.24 \pm 27.82$ & $29.63 \pm 24.54$ & 0.942 \\
Comorbid disease & & & \\
$\quad$ Diabetes mellitus, $\mathrm{n}(\%)$ & $7(23.3)$ & $9(27.3)$ & 0.720 \\
Hypertension, $\mathrm{n}(\%)$ & $23(76.7)$ & $30(90.9)$ & 0.172 \\
Coronary heart disease, $\mathrm{n}(\%)$ & $\mathrm{I}(3.3)$ & $1(3)$ & 1.000 \\
Gout, $\mathrm{n}(\%)$ & $\mathrm{I}(3.3)$ & $2(6.1)$ & 1.000 \\
$\quad$ Dyslipidemia, $\mathrm{n}(\%)$ & $16(53.3)$ & $16(48.5)$ & 0.802 \\
Body weight $(\mathrm{kg})$ & $61.1 \pm 13.1$ & $59.94 \pm 10.06$ & 0.694 \\
Body mass index $\left(\mathrm{kg} / \mathrm{m}^{2}\right)$ & $23.44 \pm 4.02$ & $23.15 \pm 3.63$ & 0.760 \\
\hline
\end{tabular}

Notes: Data presented as mean \pm SD unless indicated otherwise. Comparisons between treatment groups were done using independent $t$-test (continuous variables) and chi-square test (categorical variables).

Abbreviation: EPO, erythropoietin.

Table 2 Mean hemoglobin and hematocrit after 24 weeks of treatment

\begin{tabular}{|c|c|c|c|c|}
\hline & $\begin{array}{l}\text { Standard liquid EPO } \\
(n=30)\end{array}$ & $\begin{array}{l}\text { Lyophilized powder EPO } \\
(n=33)\end{array}$ & $\begin{array}{l}\text { Mean difference of } \\
\text { between groups }(95 \% \mathrm{Cl})\end{array}$ & $p$-value \\
\hline \multicolumn{5}{|c|}{ Hemoglobin (g/dL) } \\
\hline Baseline & $\mid I .1 \pm 0.7$ & $11.2 \pm 0.9$ & $-0.07(-0.48,0.35)$ & 0.753 \\
\hline Week 4 & $11.0 \pm 0.9$ & $10.9 \pm 1.0$ & $0.05(-0.43,0.54)$ & 0.826 \\
\hline Week 8 & $\mathrm{II} . \mathrm{I} \pm \mathrm{I} .4$ & $10.9 \pm 1.2$ & $0.17(-0.49,0.83)$ & 0.607 \\
\hline Week 12 & $10.9 \pm 1.7$ & $10.5 \pm 1.2$ & $0.4 \mathrm{I}(-0.32, \mathrm{I} .14)$ & 0.263 \\
\hline Week 16 & $\mathrm{I} \mid .4 \pm \mathrm{I} .2$ & $10.8 \pm 1.4$ & $0.55(-0.10,1.21)$ & 0.096 \\
\hline Week 20 & $10.9 \pm 1.1$ & $10.7 \pm 1.4$ & $0.18(-0.44,0.8 \mathrm{I})$ & 0.557 \\
\hline Week 24 & $10.5 \pm 1.1$ & $10.5 \pm 1.4$ & $0.02(-0.62,0.66)$ & 0.950 \\
\hline$p$-value & 0.151 & 0.057 & & 0.331 \\
\hline \multicolumn{5}{|c|}{ Hematocrit (\%) } \\
\hline Baseline & $34.3 \pm 2.4$ & $34.3 \pm 3.3$ & $-0.06(-1.51,1.39)$ & 0.934 \\
\hline Week 4 & $33.8 \pm 3.3$ & $33.7 \pm 3.3$ & $0.15(-1.50,1.8 I)$ & 0.853 \\
\hline Week 8 & $34.0 \pm 4.6$ & $33.4 \pm 3.8$ & $0.65(-1.46,2.75)$ & $0.54 I$ \\
\hline Week 12 & $33.7 \pm 5.2$ & $32.4 \pm 3.8$ & $1.31(-1.01,3.64)$ & $0.26 \mathrm{I}$ \\
\hline Week 16 & $35.2 \pm 3.6$ & $33.2 \pm 4.5$ & $2.00(-0.07,4.08)$ & 0.058 \\
\hline Week 20 & $33.7 \pm 3.4$ & $33.4 \pm 4.1$ & $0.33(-1.57,2.23)$ & 0.732 \\
\hline Week 24 & $32.5 \pm 3.6$ & $32.0 \pm 4.1$ & $0.43(-1.53,2.39)$ & 0.665 \\
\hline$p$-value & 0.248 & 0.075 & & 0.246 \\
\hline
\end{tabular}

Notes: Data presented as mean \pm SD. Comparisons between treatment groups were done using independent $t$-test (continuous variables) and comparisons within groups using the repeated measures analysis of variance.

Abbreviations: $\mathrm{Cl}$, confidence interval; EPO, erythropoietin.

increased and serum calcium decreased during lyophilized powder EPO alfa treatment, but there were no significant changes in patients continuing standard liquid EPO alfa treatment. However, no clear difference in all metabolic and biochemical parameters across the treatment arms in either study was detected (Table 4).

\section{Quality of life}

Quality of life during the study was measured. With regard to the SF-36 scores, both physical and mental components did not significantly change from baseline in both groups. The differences in the changes in the SF-36 physical and mental scores between the two groups did not reach statistical significance (Table 5).

\section{Safety profiles}

Overall, adverse events were reported by 8 of $30(26 \%)$ patients using standard liquid EPO alfa and 10 of $33(30 \%)$ patients using lyophilized powder EPO alfa. A similar frequency of adverse events was noted in the two groups. The most common adverse events that occurred in more than $10 \%$ of patients were intradialytic hypotension $(15 \%)$, myalgia (13\%) and muscle cramp (12\%). The investigators considered most adverse events to be unrelated to 

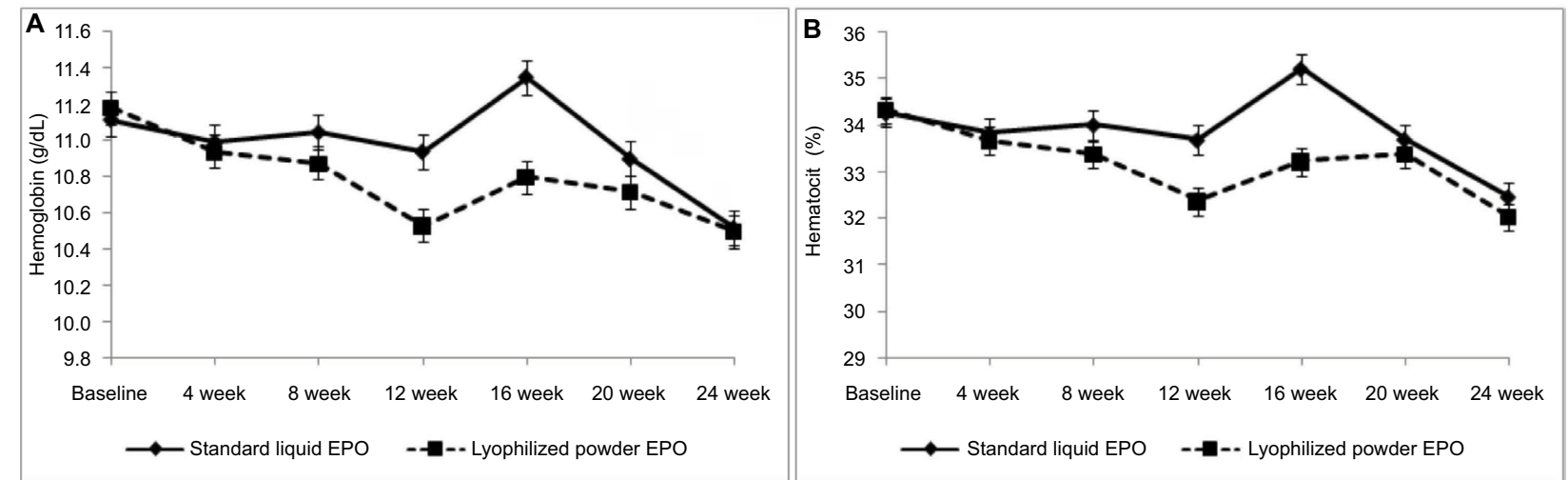

Figure 2 Observed mean \pm SD of hemoglobin $(\mathbf{A})$ and hematocrit $(\mathbf{B})$ concentrations over the study period during subcutaneous administration of lyophilized powder and standard liquid EPO alfa in the hemodialysis patients.

Abbreviation: EPO, erythropoietin.

Table 3 Mean EPO alfa dose and iron parameters after 24 weeks of treatment

\begin{tabular}{|c|c|c|c|}
\hline Assessment & $\begin{array}{l}\text { Standard liquid EPO } \\
(n=30)\end{array}$ & $\begin{array}{l}\text { Lyophilized powder EPO } \\
(n=33)\end{array}$ & $\begin{array}{l}P \text {-value } \\
\text { (EPO dose between } \\
\text { two groups) }\end{array}$ \\
\hline \multicolumn{4}{|c|}{ EPO dose (IU/kg/week) } \\
\hline Baseline & $126.4(8|.6-| 63.6)$ & II 6.9 (76.5-I44.I) & 0.531 \\
\hline Week 4 & I $25.7(80.0-168.5)$ & $124.7(76.2-154.7)$ & 0.630 \\
\hline Week 8 & $124.8(70.7-169.5)$ & $121.8(98.4-\mid 50.1)$ & 0.630 \\
\hline Week 12 & $122.6(78.3-150.4)$ & $110.3(68.9-140.6)$ & 0.536 \\
\hline Week 16 & I23.I (74.8-|45.9) & $106.1(68.9-143.4)$ & 0.441 \\
\hline Week 20 & II 8.5 (73.4-I63.9) & $108.4(70.6-145.7)$ & 0.466 \\
\hline Week 24 & $103.3(68.9-148.2)$ & $108.4(7|.8-| 54.7)$ & 0.715 \\
\hline$p$-value (within group) & 0.385 & 0.822 & \\
\hline \multicolumn{4}{|c|}{ Serum ferritin $(\mathrm{ng} / \mathrm{mL})$} \\
\hline Baseline & $490.8(243-818.1)$ & $317.2(125.2-660.3)$ & 0.740 \\
\hline Week 4 & $466.7(200.9-800.9)$ & $289.4(162-698.1)$ & 0.838 \\
\hline Week 8 & $516.8(208.8-657.6)$ & $281.4(\mid 22.8-577.2)$ & 0.491 \\
\hline Week 12 & $429.7(215.8-727.2)$ & $365.3(158.6-830.5)$ & 0.922 \\
\hline Week 16 & $355.9(225.6-772.6)$ & $420.6(125-767.3)$ & 0.846 \\
\hline Week 20 & $444.2(220.4-730.5)$ & $354.4(127.7-639.4)$ & 0.39 \\
\hline Week 24 & $512.3(207.9-733.4)$ & $356.8(I 24.1-730.4)$ & 0.401 \\
\hline$p$-value & 0.426 & 0.277 & 0.201 \\
\hline \multicolumn{4}{|c|}{ Transferrin saturation (\%) } \\
\hline Baseline & $37.8 \pm 20.4$ & $33.6 \pm 15.2$ & 0.351 \\
\hline Week 4 & $32.0 \pm 12.2$ & $34.9 \pm 19.1$ & 0.489 \\
\hline Week 8 & $29.4 \pm 12.3$ & $31.4 \pm 15.4$ & 0.569 \\
\hline Week 12 & $26.7 \pm 7.1$ & $31.7 \pm 12.3$ & 0.055 \\
\hline Week 16 & $29.6 \pm 8.8$ & $31.6 \pm 12.3$ & 0.451 \\
\hline Week 20 & $29.1 \pm 9.9$ & $33.2 \pm 12.1$ & 0.159 \\
\hline Week 24 & $34.1 \pm 13.7$ & $32.4 \pm 10.3$ & 0.583 \\
\hline$p$-value & 0.232 & 0.767 & 0.438 \\
\hline
\end{tabular}

Notes: Data presented as mean \pm SD and median (interquartile range). Comparisons between treatment groups using were done independent $t$-test and Mann-Whitney $U$ test. Comparisons within groups were done using the repeated measures analysis of variance.

Abbreviation: EPO, erythropoietin.

treatment. No deaths and serious events were reported during the study. In addition, serum antibodies against EPO at baseline and in 24-week treatment period were not found in all patients.

\section{Discussion}

This study evaluated the efficacy and safety of lyophilized powder EPO alfa among patients on hemodialysis by SC administration. Overall, results of this study showed that 
Table 4 Mean metabolic profiles after 24 weeks of treatment

\begin{tabular}{|c|c|c|c|c|}
\hline & $\begin{array}{l}\text { Standard liquid } \\
\text { EPO }(n=30)\end{array}$ & $\begin{array}{l}\text { Lyophilized powder } \\
\text { EPO }(n=33)\end{array}$ & $\begin{array}{l}\text { Mean difference of between } \\
\text { groups }(95 \% \mathrm{Cl})\end{array}$ & $p$-value \\
\hline \multicolumn{5}{|c|}{ Systolic blood pressure $(\mathrm{mmHg})$} \\
\hline Baseline & $136.4 \pm 24.8$ & $135.6 \pm 20.5$ & $0.79(-10.62,12.20)$ & 0.890 \\
\hline Week 4 & $|38| \pm 20.2$. & $138.5 \pm 17.6$ & $-0.38(-9.91,9.15)$ & 0.936 \\
\hline Week 8 & $|39.8 \pm 2| .3$ & $\mid 37.6 \pm 20.1$ & $2.19(-8.22,12.6)$ & 0.675 \\
\hline Week 12 & $|4| .5 \pm 24 . \mid$ & $139.2 \pm 20.7$ & $2.32(-8.98,13.61)$ & 0.683 \\
\hline Week 16 & $141.9 \pm 19.7$ & $|4| .8 \pm 18 . \mid$ & $0.12(-9.4,9.63)$ & 0.981 \\
\hline Week 20 & $145.9 \pm 21.6$ & $142.1 \pm 20.4$ & $3.78(-6.8,14.36)$ & 0.478 \\
\hline Week 24 & $138.0 \pm 20.9$ & $138.5 \pm \mid 8.7$ & $-0.42(-10.4,9.55)$ & 0.933 \\
\hline$p$-value & 0.260 & 0.203 & & 0.756 \\
\hline \multicolumn{5}{|c|}{ Diastolic blood pressure $(\mathrm{mmHg})$} \\
\hline Baseline & $74.5 \pm 14.4$ & $73.7 \pm 9.4$ & $0.80(-5.4 \mathrm{I}, 7.0 \mathrm{I})$ & 0.797 \\
\hline Week 4 & $76.1 \pm 12.1$ & $75.4 \pm I I .4$ & $0.64(-5.26,6.55)$ & 0.829 \\
\hline Week 8 & $76.9 \pm 12.1$ & $75.0 \pm 10.2$ & $1.90(-3.73,7.53)$ & 0.502 \\
\hline Week 12 & $77.9 \pm 12.5$ & $75.6 \pm 10.3$ & $2.33(-3.4 I, 8.06)$ & 0.420 \\
\hline Week 16 & $75.3 \pm 8.5$ & $76.9 \pm 13.3$ & $-1.52(-7.21,4.18)$ & 0.597 \\
\hline Week 20 & $77.9 \pm 11.6$ & $75.7 \pm 9.0$ & $2.27(-3.02,7.56)$ & 0.394 \\
\hline Week 24 & $72.3 \pm 12.5$ & $74.2 \pm 7.4$ & $-1.95(-7.22,3.33)$ & 0.462 \\
\hline$p$-value & 0.567 & 0.559 & & $0.77 \mathrm{I}$ \\
\hline \multicolumn{5}{|c|}{ BUN (mg/dL) } \\
\hline Baseline & $45.9 \pm 20.3$ & $45.9 \pm 15.9$ & $-0.04(-9.2,9.1)$ & 0.993 \\
\hline Week 4 & $43.7 \pm 15.4$ & $45.8 \pm 15.1$ & $-2.1(-9.8,5.6)$ & 0.587 \\
\hline Week 8 & $44.5 \pm 18.6$ & $44.5 \pm 12.5$ & $0.07(-7.9,7.9)$ & 0.986 \\
\hline Week 12 & $44.1 \pm 18.0$ & $47.0 \pm 15.6$ & $-2.9(-11.5,5.5)$ & 0.484 \\
\hline Week 16 & $47.1 \pm 19.1$ & $48.9 \pm 14.9$ & $-1.8(-10.4,6.8)$ & 0.679 \\
\hline Week 20 & $49.3 \pm 22.9$ & $50.9 \pm 16.5$ & $-1.6(-11.8,8.6)$ & $0.75 \mathrm{I}$ \\
\hline Week 24 & $49.5 \pm 17.2$ & $50.2 \pm 18.2$ & $-0.7(-9.6,8.3)$ & 0.882 \\
\hline$p$-value & 0.094 & 0.707 & & 0.695 \\
\hline \multicolumn{5}{|c|}{ Creatinine (mg/dL) } \\
\hline Baseline & $8.7 \pm 3.1$ & $8.7 \pm 2.3$ & $-0.04(-1.4,1.3)$ & 0.949 \\
\hline Week 4 & $8.6 \pm 2.7$ & $8.7 \pm 2.4$ & $-0.1(-1.4,1.2)$ & 0.861 \\
\hline Week 8 & $8.3 \pm 2.7$ & $8.9 \pm 2.6$ & $-0.7(-1.9,0.7)$ & 0.338 \\
\hline Week 12 & $8.7 \pm 3.2$ & $8.6 \pm 2.3$ & $0.1(-1.3,1.5)$ & 0.856 \\
\hline Week 16 & $8.5 \pm 2.7$ & $9.1 \pm 2.4$ & $-0.5(-1.8,0.8)$ & 0.414 \\
\hline Week 20 & $8.9 \pm 3.3$ & $9.6 \pm 2.3$ & $-0.7(-2.1,0.8)$ & 0.347 \\
\hline Week 24 & $9.2 \pm 2.8$ & $9.5 \pm 2.4$ & $-0.3(-1.6,1.0)$ & 0.655 \\
\hline$p$-value & 0.150 & 0.036 & & 0.521 \\
\hline \multicolumn{5}{|c|}{ Serum albumin (g/dL) } \\
\hline Baseline & $4.2 \pm 0.4$ & $4 . I \pm 0.4$ & $0.1(-0.1,0.3)$ & 0.398 \\
\hline Week 4 & $4.1 \pm 0.4$ & $4.0 \pm 0.5$ & $0.1(-0.2,0.3)$ & 0.650 \\
\hline Week 8 & $4.1 \pm 0.5$ & $4.1 \pm 0.5$ & $-0.04(-0.3,0.2)$ & 0.773 \\
\hline Week 12 & $4.0 \pm 0.5$ & $4.1 \pm 0.4$ & $-0.02(-0.3,0.2)$ & 0.856 \\
\hline Week 16 & $4.2 \pm 0.5$ & $4.2 \pm 0.3$ & $0.01(-0.2,0.2)$ & 0.934 \\
\hline Week 20 & $4.1 \pm 0.4$ & $4.2 \pm 0.4$ & $-0.08(-0.3,0.1)$ & 0.392 \\
\hline Week 24 & $4.1 \pm 0.4$ & $4.1 \pm 0.4$ & $-0.06(-0.3,0.2)$ & 0.569 \\
\hline$p$-value & 0.605 & 0.107 & & 0.947 \\
\hline \multicolumn{5}{|c|}{ Serum calcium (mg/dL) } \\
\hline Baseline & $9.3 \pm 0.7$ & $9.4 \pm 0.9$ & $-0.1(-0.5,0.3)$ & 0.623 \\
\hline Week 4 & $9.1 \pm 0.9$ & $8.9 \pm 0.8$ & $0.1(-0.3,0.6)$ & 0.513 \\
\hline Week 8 & $9.3 \pm 1.1$ & $9.1 \pm 0.8$ & $0.2(-0.2,0.7)$ & 0.318 \\
\hline Week 12 & $9.1 \pm 0.7$ & $9.2 \pm 0.7$ & $-0.1(-0.5,0.3)$ & 0.649 \\
\hline Week 16 & $9.5 \pm 1.3$ & $9.1 \pm 0.8$ & $0.4(-0.2,0.9)$ & 0.185 \\
\hline Week 20 & $9.1 \pm 0.6$ & $9.1 \pm 0.8$ & $0.03(-0.4,0.4)$ & 0.877 \\
\hline Week 24 & $9.2 \pm 0.9$ & $8.9 \pm 0.6$ & $0.2(-0.1,0.6)$ & 0.198 \\
\hline$p$-value & 0.662 & $0.040^{*}$ & & 0.406 \\
\hline
\end{tabular}


Table 4 (Continued)

\begin{tabular}{|c|c|c|c|c|}
\hline & $\begin{array}{l}\text { Standard liquid } \\
\text { EPO }(n=30)\end{array}$ & $\begin{array}{l}\text { Lyophilized powder } \\
\text { EPO }(n=33)\end{array}$ & $\begin{array}{l}\text { Mean difference of between } \\
\text { groups }(95 \% \mathrm{Cl})\end{array}$ & $p$-value \\
\hline \multicolumn{5}{|c|}{ Serum phosphorus (mg/dL) } \\
\hline Baseline & $4.3 \pm 1.2$ & $4.9 \pm 1.6$ & $-0.6(-1.3,0.1)$ & 0.110 \\
\hline Week 4 & $4.2 \pm 1.1$ & $5.1 \pm 1.9$ & $-0.9(-1.7,-0.2)$ & 0.017 \\
\hline Week 8 & $4.1 \pm 1.2$ & $4.8 \pm 1.4$ & $-0.7(-1.4,-0.1)$ & 0.035 \\
\hline Week 12 & $4.4 \pm 1.6$ & $4.6 \pm 1.7$ & $-0.2(-1.0,0.7)$ & 0.658 \\
\hline Week 16 & $4.5 \pm 1.4$ & $4.8 \pm 1.4$ & $-0.2(-0.9,0.5)$ & 0.525 \\
\hline Week 20 & $4.6 \pm 1.8$ & $5.2 \pm 1.4$ & $-0.7(-1.5,0.1)$ & 0.089 \\
\hline Week 24 & $4.9 \pm 1.9$ & $4.8 \pm 1.5$ & $0.1(-0.8,0.9)$ & 0.786 \\
\hline$p$-value & 0.059 & 0.989 & & 0.079 \\
\hline \multicolumn{5}{|c|}{ Intact PTH (pg/mL) } \\
\hline Baseline & $194.4 \pm 159.3$ & $211.1 \pm 157.9$ & $-16.7(-96.7,63.3)$ & 0.677 \\
\hline Week 4 & $230.4 \pm 266.9$ & $249.7 \pm 137.7$ & $-19.3(-128.9,90.3)$ & 0.725 \\
\hline Week 8 & $227.6 \pm 191.4$ & $213.9 \pm 149.2$ & | $3.6(-79.4,106.7)$ & 0.770 \\
\hline Week 12 & $273.3 \pm 260.3$ & $231.7 \pm 173.3$ & $41.6(-68.9, \mid 152.1)$ & 0.454 \\
\hline Week 16 & $299.0 \pm 319.7$ & $251.2 \pm 195.5$ & $47.9(-88.6,184.3)$ & 0.484 \\
\hline Week 20 & $308.3 \pm 347.5$ & $254.1 \pm 2 \mid 4.2$ & $54.2(-89.8,198.2)$ & 0.455 \\
\hline Week 24 & $331.9 \pm 291.7$ & $324.5 \pm 311.3$ & $7.4(-157.6,172.5)$ & 0.928 \\
\hline$p$-value & $0.038 *$ & 0.191 & & 0.662 \\
\hline
\end{tabular}

Notes: Data presented as mean \pm SD. Comparisons between treatment groups were done using independent $t$-test (continuous variables) and comparisons within groups were done using the repeated measures analysis of variance.

Abbreviations: $\mathrm{Cl}$, confidence interval; EPO, erythropoietin; BUN, blood urea nitrogen; PTH, parathyroid hormone.

Table 5 Quality of life using the SF-36 questionnaire after 24 weeks of treatment

\begin{tabular}{llll}
\hline Assessment & $\begin{array}{l}\text { Standard liquid EPO } \\
(\mathbf{n}=\mathbf{3 0})\end{array}$ & $\begin{array}{l}\text { Lyophilized powder EPO } \\
(\mathbf{n}=\mathbf{3 3})\end{array}$ & $\begin{array}{l}\mathbf{p} \text {-value } \\
\text { (EPO dose between two groups) }\end{array}$ \\
\hline Physical health score & & & 0.911 \\
Baseline & $63.87 \pm 22.24$ & $63.27 \pm 19.56$ & 0.425 \\
Week 24 & $60.4 \pm 27.47$ & $65.52 \pm 23.06$ & \\
p-value (within group) & 0.404 & 0.519 & 0.362 \\
Mental health score & & & 0.364 \\
Baseline & $66.23 \pm 19.99$ & $70.67 \pm 18.34$ & \\
Week 24 & $65.77 \pm 27.96$ & $71.79 \pm 24.29$ & \\
p-value (within group) & 0.920 & 0.765 & 0.726 \\
Total score & & & 0.348 \\
Baseline & $67.47 \pm 21.88$ & $69.27 \pm 18.81$ & \\
Week 24 & $65.5 \pm 28.66$ & $71.79 \pm 24.07$ & \\
p-value (within group) & 0.655 & 0.498 & \\
\hline
\end{tabular}

Notes: Data presented as mean \pm SD. Comparisons between treatment groups were done using independent $t$-test (continuous variables) and comparisons within groups were done using the repeated measures analysis of variance.

Abbreviation: EPO, erythropoietin.

SC administration of lyophilized powder EPO alfa for 24 weeks exhibited equivalent efficacy and safety as standard liquid EPO alfa regarding hemoglobin maintenance for anemia treatment in this patient population. No statistically significant difference in mean weekly dose of EPO alfa was observed between the two groups. Overall, differences between formulations were not statistically significant for any of the calculated parameters.

Limited clinical studies have reported on lyophilized powder EPO alfa treatment. ${ }^{11,12}$ The first study among malignancy patients showed that $64.7 \%$ had hemoglobin normalization and blood transfusions were reduced by $50 \% .{ }^{11}$ In the second study, a randomized controlled investigation was carried out among patients with CKD on hemodialysis. The lyophilized powder EPO alfa was effective and safe the same as the standard reference EPO alfa over 24 weeks when administered intravenously for hemoglobin maintenance among patients on hemodialysis. ${ }^{12}$ Based on our results and related studies, SC lyophilized powder EPO alfa injection appeared to be effective and comparable to standard liquid EPO alfa in maintaining hemoglobin levels among patients with end-stage renal disease receiving hemodialysis. A stable hemoglobin 
level was maintained in more than $90 \%$ of patients without any increase of the EPO dose. In individuals with higher baseline EPO alfa dose requirements, a somewhat higher lyophilized powder EPO alfa dose requirement was noted. A study of longer duration is needed to determine the durability of response among such patients.

The efficacy of EPO alfa administration can be affected by iron metabolism, nutritional and metabolic factors and functional status of patients. ${ }^{13}$ For this reason, the iron status, nutritional parameters, intact PTH and quality of life were monitor every 4 weeks in our study and intravenous iron was supplied. No significant difference was found between the groups for these parameters during the study. It might be expected that SC administration of lyophilized powder EPO alfa was as effective as standard liquid EPO alfa in maintaining stable hemoglobin levels. The economical benefit is also appreciated in Thailand because the cost per unit of lyophilized powder EPO alfa preparation is lower than that of standard liquid EPO alfa.

EPO by SC administration is more convenient than intravenous administration in CKD patients without hemodialysis. Treatment with EPO SC injection produced higher EPO levels and improved efficiency, resulting in a reduced dosing requirement for EPO by $33 \%$ to maintain the target hemoglobin level. ${ }^{14-16}$ However, some studies found that three times weekly intravenous or SC EPO did not differ in the total monthly erythropoietin doses and average monthly hemoglobin levels. ${ }^{17,18}$ In the present study, the EPO doses used for maintaining hemoglobin levels also did not significantly differ when switching from intravenous to SC injection. However, SC administration was one of the associated factors with the development of anti-EPO antibodies. Therefore, the balance of the risk of immunogenicity and injection pain associated with SC administration in combination with other potential benefits of improved EPO response and no EPO handing due to SC injected by themselves should be considered.

Observations from our study suggest no obvious safety concerns because the adverse events reported were consistent with the dialysis patient population. Many adverse events were observed during hemodialysis sessions. During the follow-up period, very few and only mild EPO-related adverse events were noted. Recently, a significant higher incidence of the EPO-induced pure red cell aplasia (PRCA) was reported. ${ }^{19,20}$ This finding has been related to packaging and storage conditions and using different brands of biosimilar EPO agents. ${ }^{20-23}$ Most PRCA patients received EPO alfa by SC route that could increase immunogenicity. ${ }^{24}$ In our study, antibodies against EPO were investigated in all patients; the titer of serum anti-EPO antibody level was negative and no case of EPO-induced PRCA was detected. The benefits of lyophilized powder EPO alfa in EPO-induced PRCA could be the cost savings due to the decreased expense for cold-chain distribution. Notably, this study was a short-term follow-up study for only 24 weeks to derive any conclusion on the immunogenicity of the medication.

This study had some limitations. Because of the short duration, it did not assess whether the effects of lyophilized powder EPO alfa on hemoglobin could be sustained over a longer period. The outcomes of this study were based on a relatively small sample of primarily Asian patients and may not be generalizable to a broader population. Compliance was assessed by returned injection chart record, which may have overestimated compliance rates. However, the patient demographic data were consistent with the general hemodialysis population with high comorbid illness. Hence, the study population was representative of patients on hemodialysis and treated for renal anemia in clinical practice. Concerning short-term safety profiles, the introduction of biosimilar EPO agents for practical use remains a controversial issue regarding current treatment. Differences in producing molecules and/or manufacturing processes have created small differences in the composition of recombinant human EPO formulation. Therefore, clinical and immunogenicity monitoring in terms of long-term safety or efficacy is required.

In conclusion, among patients receiving hemodialysis and then switching from standard liquid EPO alfa to SC lyophilized powder EPO alfa injection, mean hemoglobin concentrations for 24 weeks were effectively maintained. In this study, the effect of SC lyophilized powder EPO alfa on hemoglobin level was noted in the absence of clinically serious adverse events including EPO-induced PRCA. The safety of SC lyophilized powder EPO alfa injection in PRCA will require further evaluation in longer duration studies.

\section{Acknowledgments}

The authors wish to acknowledge the contributions of the following individuals to this study: the nurses in the Dialysis Center and staff in the Biomedical Clinical Research Center in Phramongkutklao Hospital, Bangkok, Thailand and Chakkraphong Hospital, Prachinburi, Thailand. This work was supported by a grant from the Phramongkutklao Hospital and College of Medicine, the National Science and Technology Development Agency (NSTDA, P-13-00505), and Berli Jucker Public Company Limited, Bangkok, Thailand.

\section{Disclosure}

The authors report no conflicts of interest in this work. 


\section{References}

1. Babitt JL, Lin HY. Mechanisms of anemia in CKD. J Am Soc Nephrol. 2012;23(10):1631-1634.

2. McGonigle RJ, Wallin JD, Shadduck RK, Fisher JW. Erythropoietin deficiency and inhibition of erythropoiesis in renal insufficiency. Kidney Int. 1984;25(2):437-444.

3. Koch KM, Frei U. Treatment of renal anemia, 1960-1990. Adv Nephrol Necker Hosp. 1991;20:19-30.

4. Weiss G, Goodnough LT. Anemia of chronic disease. $N$ Engl J Med. 2005;352(10):1011-1023.

5. Aronoff GR, Duff DR, Sloan RS, et al. The treatment of anemia with low-dose recombinant human erythropoietin. Am J Nephrol. 1990;10(Suppl 2):40-43.

6. Cody J, Daly C, Campbell M, et al. Recombinant human erythropoietin for chronic renal failure anaemia in pre-dialysis patients. Cochrane Database Syst Rev. 2005;(3):CD003266.

7. Cody JD, Hodson EM. Recombinant human erythropoietin versus placebo or no treatment for the anaemia of chronic kidney disease in people not requiring dialysis. Cochrane Database Syst Rev. 2016;(1):CD003266.

8. Foley RN, Curtis BM, Parfrey PS. Erythropoietin therapy, hemoglobin targets, and quality of life in healthy hemodialysis patients: a randomized trial. Clin J Am Soc Nephrol. 2009;4(4):726-733.

9. Geng Y, Yuan W, Wu F, Chen J, He M, Jin T. Formulating erythropoietinloaded sustained-release PLGA microspheres without protein aggregation. J Control Release. 2008;130(3):259-265.

10. Donato H, Vain N, Rendo P, et al. Effect of early versus late administration of human recombinant erythropoietin on transfusion requirements in premature infants: results of a randomized, placebo-controlled, multicenter trial. Pediatrics. 2000;105(5):1066-1072.

11. Lastiri JM, Specterman SR, Rendo P, Pallotta MG, Varela MS, Goldstein $\mathrm{S}$. [Predictive response variables to recombinant human erythropoietin treatment in patients with anemia and cancer]. Medicina (B Aires). 2002;62(1):41-47. Spanish [with English abstract].

12. Satirapoj B, Supasyndh O, Choovichian P. A comparative study of efficacy and safety of the lyophilized powder alpha-erythropoietin and the liquid form alpha-erythropoietin for hemoglobin maintenance in patients with hemodialysis treatment. J Med Assoc Thai. 2014;97(9):899-906.
13. Lankhorst CE, Wish JB. Anemia in renal disease: diagnosis and management. Blood Rev. 2010;24(1):39-47.

14. Besarab A, Reyes CM, Hornberger J. Meta-analysis of subcutaneous versus intravenous epoetin in maintenance treatment of anemia in hemodialysis patients. Am J Kidney Dis. 2002;40(3):439-446.

15. Kaufman JS, Reda DJ, Fye CL, et al. Subcutaneous compared with intravenous epoetin in patients receiving hemodialysis. Department of Veterans Affairs Cooperative Study Group on Erythropoietin in Hemodialysis Patients. N Engl J Med. 1998;339(9):578-583.

16. Paganini EP, Eschbach JW, Lazarus JM, et al. Intravenous versus subcutaneous dosing of epoetin alfa in hemodialysis patients. Am J Kidney Dis. 1995;26(2):331-340.

17. Jensen JD, Madsen JK, Jensen LW. Comparison of dose requirement, serum erythropoietin and blood pressure following intravenous and subcutaneous erythropoietin treatment of dialysis patients. IV and SC erythropoietin. Eur J Clin Pharmacol. 1996;50(3):171-177.

18. De Schoenmakere G, Lameire N, Dhondt A, et al. The haematopoietic effect of recombinant human erythropoietin in haemodialysis is independent of the mode of administration (i.v. or s.c.). Nephrol Dial Transplant. 1998;13(7):1770-1775.

19. Rossert J; Pure Red Cell Aplasia Global Scientific Advisory Board (GSAB). Erythropoietin-induced, antibody-mediated pure red cell aplasia. Eur J Clin Invest. 2005;35(Suppl 3):95-99.

20. Praditpornsilpa K, Tiranathanagul K, Kupatawintu P, et al. Biosimilar recombinant human erythropoietin induces the production of neutralizing antibodies. Kidney Int. 2011;80(1):88-92.

21. Wish JB. Erythropoiesis-stimulating agents and pure red-cell aplasia: you can't fool Mother Nature. Kidney Int. 2011;80(1):11-13.

22. Weber G, Gross J, Kromminga A, Loew HH, Eckardt KU. Allergic skin and systemic reactions in a patient with pure red cell aplasia and anti-erythropoietin antibodies challenged with different epoetins. $\mathrm{JAm}$ Soc Nephrol. 2002;13(9):2381-2383.

23. Bennett CL, Luminari S, Nissenson AR, et al. Pure red-cell aplasia and epoetin therapy. N Engl J Med. 2004;351(14):1403-1408.

24. McKoy JM, Stonecash RE, Cournoyer D, et al. Epoetin-associated pure red cell aplasia: past, present, and future considerations. Transfusion. 2008;48(8):1754-1762.
The International Journal of Nephrology and Renovascular Disease is an international, peer-reviewed open access journal focusing on the pathophysiology of the kidney and vascular supply. Epidemiology, screening, diagnosis, and treatment interventions are covered as well as basic science, biochemical and immunological studies. The manuscript
Dovepress

management system is completely online and includes a very quick and fair peer-review system, which is all easy to use. Visit http://www. dovepress.com/testimonials.php to read real quotes from published authors. 